\title{
Modeling and Characterization for Microstrip Filters in the Manufacturing Process through the Unscented Transform and Use of Electromagnetic Simulators
}

\author{
Abraham E. Ortega Paredes, Leonardo R. A. X. de Menezes, \\ Humberto Abdalla Jr., and Ivan N. A. Romani \\ Departmento de Engenharia Elétrica, Faculdade de Tecnologia, Universidade de Brasília 70910-900, Brasília, DF, Brazil \\ Correspondence should be addressed to Leonardo R. A. X. de Menezes, leonardo@ene.unb.br
}

Received 14 December 2009; Revised 11 August 2010; Accepted 1 September 2010

Academic Editor: Andrzej Dzielinski

Copyright (c) 2010 Abraham E. Ortega Paredes et al. This is an open access article distributed under the Creative Commons Attribution License, which permits unrestricted use, distribution, and reproduction in any medium, provided the original work is properly cited.

\begin{abstract}
This paper presents the unscented transform (UT) applied to uncertainty modeling of manufacturing tolerances at the design stage of microwave passive devices. The process combines the UT with electromagnetic simulations and assumes that the numerical sources of error are negligible in comparison to the imperfections due to the manufacturing process. The technique was validated with the simulation, construction, and test of several sets of identical microstrip filters with very good results. Although the combination of UT and electromagnetic simulators was presented for microstrip filters, it can also be used for different types of microwave devices.
\end{abstract}

\section{Introduction}

Electromagnetic simulation has become an integral part in the analysis and validation of microwave devices. However, the manufacturing process can introduce errors that might degrade the actual performance. Such effects can increase the number of required prototypes and also the final production cost.

One solution to avoid these problems is to model the uncertainties in the assembly process. The most popular method for such uncertainties is the use of random variables in the simulation process with the Monte Carlo technique [1]. The main drawback of this approach is that it requires several thousands of simulations to get the statistics of the final result. Therefore, it is useful to use alternate uncertainty modeling techniques which can reduce the problem substantially. One of these approaches is the unscented transform (UT). The UT is based on the approximation of a continuous probability density function by a discrete one. Since the distribution is discrete, only a finite number of simulations will be required to capture the statistics of the problem. The use of UT allows efficient use of electromagnetic simulation in the characterization of mapped random variables. It is important to notice that the simulator response is not an exact result but an approximation of the actual answer. The use of different simulators with diverse numerical methods means that the same filter may yield slightly different responses depending on the simulator used.

\section{Theory}

2.1. Modeling Error. The manufacturing process of a filter usually introduces errors in the dimensions. If there is only one source of error, then this may be modeled by a single random variable with fixed mean and a certain probability distribution. If the actual distribution of the process was entirely characterized, then the unscented transform could also be used with greater accuracy. If more variables are also subjected to the manufacturing process, then more random variables with suitable distributions are required resulting in a greater number of simulations. In this work, the variables were considered independent and limited to two by a filter as basic simplification. 
Since an electromagnetic simulator is being used, in addition to the manufacturing error there is also the numerical error. This source of uncertainty is usually difficult to quantify or geometry dependent. For this reason its source of error was not modeled in this work.

2.2. Modeling a Continuous Distribution by a Discrete One. The characterization of uncertainty in a certain function $G(x)$ is equivalent to the problem of calculating the statistical moments of a random variable $\hat{u}$ submitted to the mapping $G(\hat{u})$. If the function $G(x)$ can be represented by its Taylor expansion, then a truncated polynomial representation can be written by

$$
G(x)=a_{0}+a_{1} x+a_{2} x^{2}+\cdots=\sum_{n=0}^{n} a_{n} x^{n} .
$$

Since the polynomial is truncated, it implies that the expected value of the mapping is

$$
E\{G(\widehat{u})\}=\int G(u) p(u) d u=E\left\{\sum_{m=0}^{k} a_{m} u^{m}\right\}=\sum_{m=0}^{k} a_{m} E\left\{u^{m}\right\},
$$

where $p(u)$ is the continuous probability density function and $\hat{u}$ is the continuous random variable. Therefore, the calculation of the expected value using a continuous distribution results is

$$
E\{G(\widehat{u})\}=\sum_{m=0}^{k} a_{m} \int u^{m} p(u) d u .
$$

If the distribution is discrete, the expected value will be

$$
E\{G(\hat{u})\}=\sum_{m=0}^{k} a_{m} \sum_{n} p_{n} u_{n}^{m}
$$

where $p_{n}$ are the weights (probabilities) of the discrete distribution and $u_{n}$ are the sigma points (discrete points of the distribution). Since the mapping has the same results for the discrete and continuous ones, then

$$
\sum_{n} G\left(u_{n}\right) p_{n}=\int G(u) p(u) d u .
$$

Equation (5) shows that the moments of the mapped variable can be calculated either using a continuous or discrete distribution. The result will be the same provided that the moments of the continuous and discrete distributions are equal. Naturally, the discrete distribution is more useful for simulation purposes since it needs calculation only at a finite number of points.

2.3. The Theory of UT. Julier and Uhlmann [2] developed the UT in 1997, and it has been used in various areas of electrical engineering $[3,4]$. The UT is completely defined once the discrete distribution mimics the continuous one. For that, it is necessary to calculate the appropriate weights $p_{n}$ and

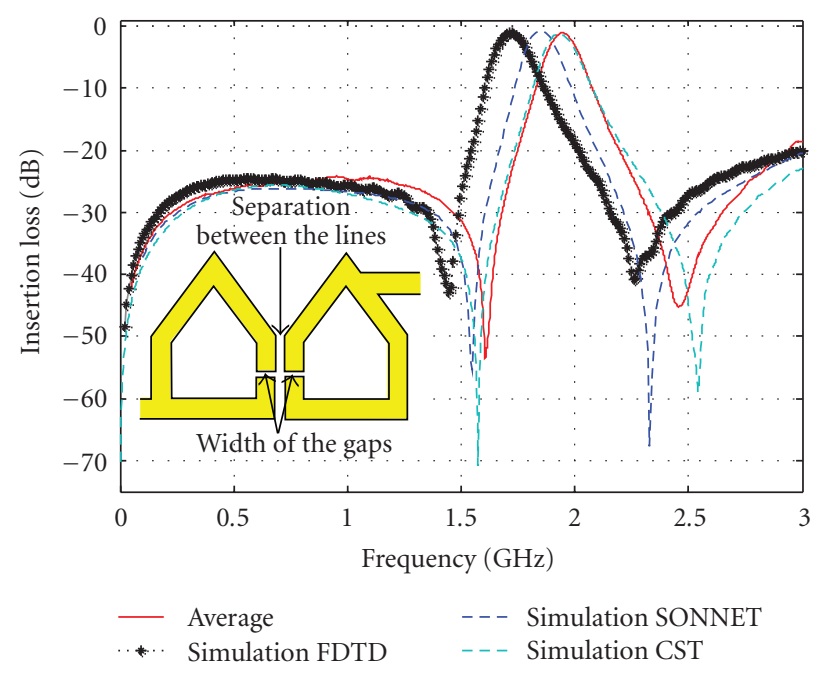

FIGURE 1: Comparison between average, FDTD, SONNET, and CST.

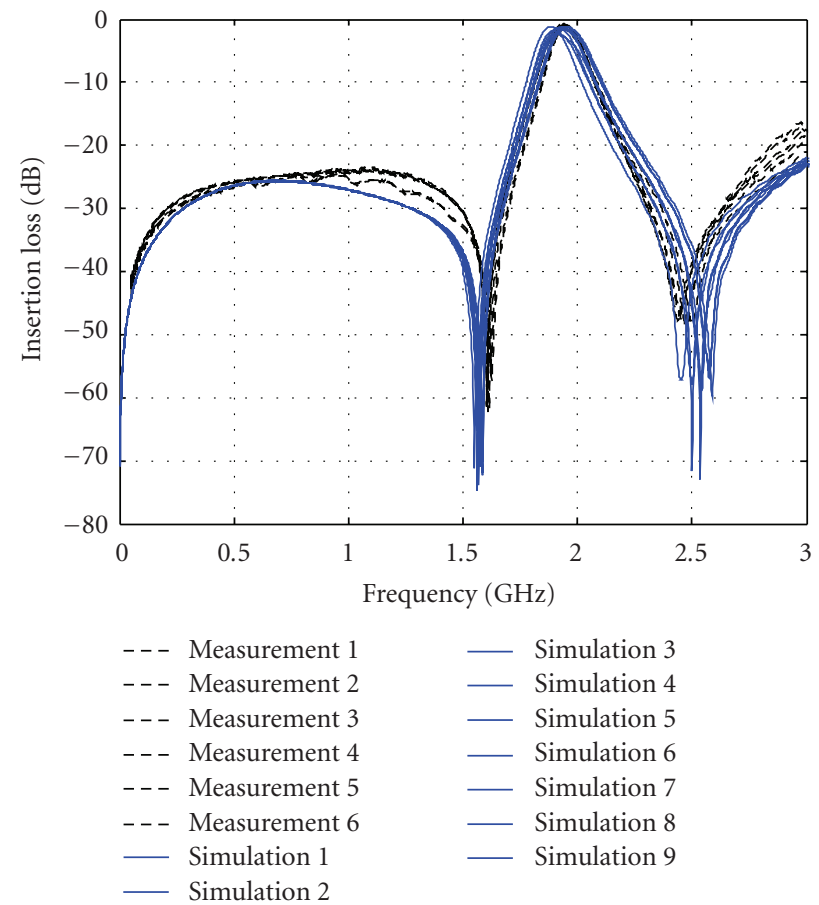

FIGURE 2: Comparison between measurement and simulation.

$u_{n}$ sigma points. Since (5) has to hold for all moments, the calculation of the weights and sigma points is the solution of a system

$$
\begin{gathered}
\sum_{i=1}^{n} u_{i} p_{i}=E\{\hat{u}\}=\int u p(u) d u, \\
\vdots \\
\sum_{i=1}^{n} u_{i}^{k} p_{i}=E\left\{\hat{u}^{k}\right\}=\int u^{k} p(u) d u .
\end{gathered}
$$




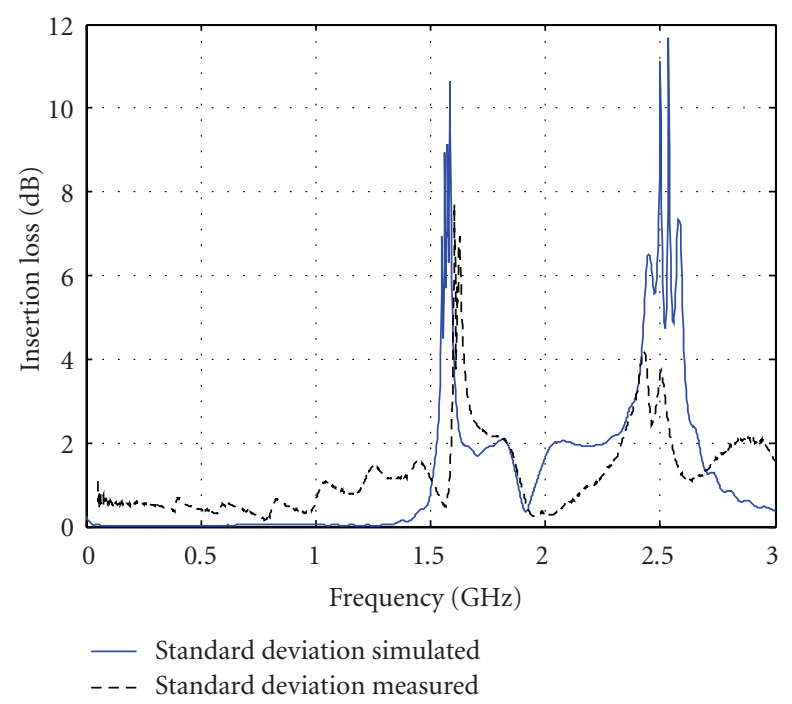

FIgURE 3: Standard deviation simulated and measured.

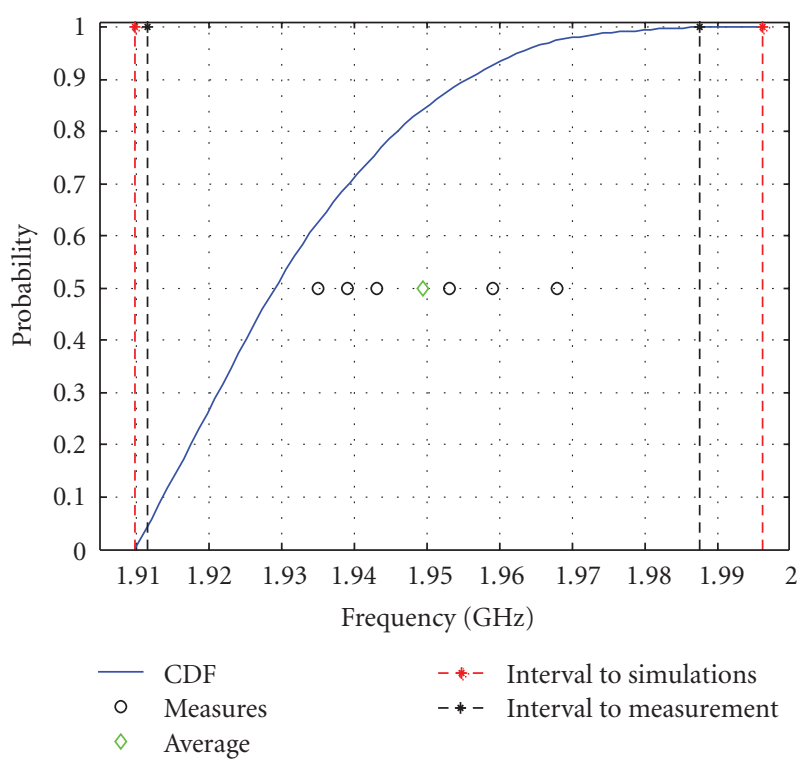

FIgURE 4: CDF of the center frequency.

This system has to be truncated at some point. A satisfactory compromise is limiting the system up to the fourth moment. Another useful simplification is to consider that the distribution has zero mean and unitary variance.

If $u$ is a random variable with mean $U$, standard deviation $\sigma$, skewness $\gamma_{1}$, and excess kurtosis $\gamma_{2}$, the sigma points are

$$
\begin{aligned}
& S_{1}=U+\frac{1}{2}\left[\gamma_{1}-\sqrt{4\left(\gamma_{2}+3\right)-3 \gamma_{1}^{2}}\right] \sigma, \\
& S_{2}=U+\frac{1}{2}\left[\gamma_{1}+\sqrt{4\left(\gamma_{2}+3\right)-3 \gamma_{1}^{2}}\right] \sigma \\
& S_{3}=U .
\end{aligned}
$$

2.4. Applying the UT to the Uniform Distribution. The sigma points and weights are simple once the probability density

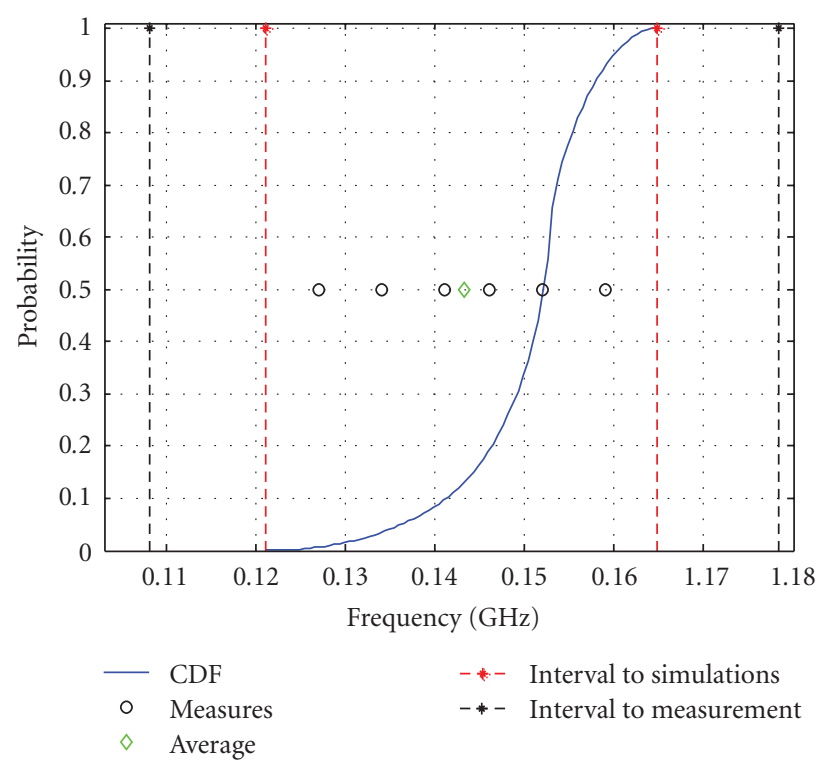

FIgURE 5: CDF to bandwidth.

TABle 1: Sigma points and weights for the normalized uniform distribution.

\begin{tabular}{ccc}
\hline$n$ & $\begin{array}{c}\text { Zeros (normalized sigma } \\
\text { points) }\end{array}$ & Weights \\
\hline 0 & 0 & 1 \\
1 & $-0.577,0.577$ & $0.5,0.5$ \\
2 & $-0.77459,0,0.77459$ & $0.278,0.444,0.278$ \\
3 & $-0.86114,-0.33998,0.33998$, & $0.1740,0.326,0,0.326,0.1740$ \\
\hline
\end{tabular}

TABLE 2: Points of sigma approach to one variable.

\begin{tabular}{lc}
\hline Number of simulations & Width of gap $(\mathrm{mm})$ \\
\hline 1 & $2.25-0.577 * 0.05=2.2211$ \\
2 & $2.25+0.577 * 0.05=2.2788$ \\
\hline
\end{tabular}

function of the source of uncertainty is known. In this work, the uniform distribution was used as the probability function. The sigma points and weights for the case of the uniform distribution in the interval $[-1,1]$ are presented in Table 1.

Table 2 shows how to combine the information of Table 1 in an electromagnetic simulation. In this example, one may consider a particular device with a single transmission line with an average width of $2.25 \mathrm{~mm}$ and an error of $0.1 \mathrm{~mm}$ (uniform distribution). The first order UT approximation ( $n=1$ in Table 1$)$ requires three simulations to characterize the statistics of the problem, as shown in Table 2.

Each sigma point of Table 2 will yield one result. This can be any number of output parameters: insertion loss, return loss, bandwidth, center frequency, and so on. The statistical parameters of the response $G(\widehat{u})$ will be calculated using

$$
E\{G(\hat{u})\}=\frac{1}{2} G(2.2211)+\frac{1}{2} G(2.2788) .
$$


Although (8) shows the calculation of the first noncentral moment of response, the UT may yield a number of statistical parameters. In this work the main ones are expected value, standard deviation, and cumulative distribution function (which allows the characterization of the confidence interval).

The UT also provides information on which variables are more relevant for variations in the response and how many random variables are needed for the model. One simple form to determine the most relevant variables is to determine the proportion of variation. If $x$ and $y$ are two variables, one may use the proportion

$$
\frac{\operatorname{Var}\{x\}}{\operatorname{Var}\{x\}+\operatorname{Var}\{y\}} \text { or } \quad \frac{\operatorname{Var}\{y\}}{\operatorname{Var}\{x\}+\operatorname{Var}\{y\}} \text {. }
$$

Equation (9) is an approximation of the relative importance between the variables. Once the solutions to the set of sigma points are calculated, the results can be combined according to the weighted average calculation of the UT theory [2].

\section{Comparison between Different Simulators}

The UT was used to model manufacturing errors at the simulator stage instead of at the prototype stage. The main assumption is that the simulator response is a very good approximation of one ideal device (without assembly errors). Unfortunately the value of the numerical uncertainty may be somewhat dependent on the problem. This assumption was verified in a microstrip second-order bandpass filter [5], designed with a center frequency of $1.8 \mathrm{GHz}$ and $150 \mathrm{MHz}$ bandwidth. The filter was implemented in microstrip technology in substrate of relative permittivity $\varepsilon_{r}=10$ and thickness $h=1.57 \mathrm{~mm}$.

These filters had six prototypes assembled using the same process based on a single design. The original designs were simulated in different simulation packages: CST, SONNETLite, and a standard FDTD simulator.

These reference simulations were then compared to the measurements of the prototypes. The purpose of the comparison was to determine which simulator had the best response in comparison to the measurements. Naturally, all programs had their strong and weak points, so a score (13 ) was devised to rank the response of each simulator (see Table 3).

The simulations were compared to the average result of the measurements of the filter. As Figure 1 shows, there is a difference between measured and simulated center frequencies. Figure 1 also allows the calculation of the filter bandwidth. As expected, the CST simulator shows a better approximation to center frequency and bandwidth rather than the other techniques.

\section{Results}

The statistical characterization consisted in obtaining the expected value, standard deviation, and cumulative probability function (CDF). The calculation of the CDF used the
TABLE 3: Score of the simulators.

\begin{tabular}{lccc}
\hline & C. Freq. & Bandwidth & Mean Arit. \\
\hline FDTD & 1 & 1 & $\mathbf{1}$ \\
SONNET & 2 & 2 & $\mathbf{2}$ \\
CST & 3 & 2 & $\mathbf{2 . 5}$ \\
\hline
\end{tabular}

techniques developed in [6]. The manufacturing technique used in this work is detailed in [5].

4.1. Sources of Uncertainty. The two clearest sources of uncertainty are the width of the gaps and the separation between the lines, as shown in Figure 1. These sources of uncertainty were characterized with two independent random variables with uniform distribution in the interval $[-0.1 \mathrm{~mm}, 0.1 \mathrm{~mm}]$.

4.2. Measurements and Simulations. Figure 2 shows the comparison between the measured and simulated responses of the filter using the respective sigma points.

Figure 3 shows the measured and calculated standard deviation. The standard deviation indicates the dispersion of the variable over the average. The smaller the standard deviation means less variation over the average.

\subsection{Cumulative Probability Function of Selected Parameters}

4.3.1. Center Frequency. The CDF of the center frequency of filter is presented in Figure 4. This figure shows an estimate of the $95 \%$ confidence intervals for measurements. All the measurements are included in the interval to simulation, as well as its average.

However, the values of measured central frequency are a clear indication that there are still sources of errors that were not modeled.

4.3.2. Bandwidth. The CDF of the bandwidth is presented in Figure 5. The curve shows that all measurement points are inside the range defined by the simulation $95 \%$ confidence interval. As well as for central frequency, some of the errors were not entirely modeled either because of the intrinsic error introduced by the simulator or perhaps because the simulator did not perform well regarding this parameter. It is unlikely that this error comes from the measurement.

\section{Conclusions}

This paper presented a simple and accurate technique for characterization of uncertainties by UT with electromagnetic simulators. This characterization allows the inclusion of the uncertainties introduced in the manufacturing process into the electromagnetic simulation. After several tests, the numerical tool that had the best performance was chosen to model the effect of uncertainties (in these cases the CST software package).

An important conclusion is that all simulators introduce some kind of intrinsic error that may, under certain 
circumstances, emerge as a dominant mechanism of the overall error. This means that even if the other sources of uncertainty are adequately modeled, there may be cases where the numerical error masks the effect of manufacturing uncertainties in the final results. Unfortunately, this error still cannot be adequately modeled using the UT.

The validation of this method was performed including the effects of uncertainty in the simulation and comparing the effects with real measurements of the filters. The results indicate that the combination of UT and EM simulator can characterize accurately the effects of uncertainty.

\section{Acknowledgment}

This work was supported by the Brazilian Research Agency (CNPq).

\section{References}

[1] Papoulis, Probability, Random Variables, and Stochastic Processes, McGraw-Hill, New York, NY, USA, 3rd edition, 1991.

[2] S. J. Julier and J. K. Uhlmann, "Unscented filtering and nonlinear estimation," Proceedings of the IEEE, vol. 92, no. 3, pp. 401-422, 2004.

[3] J. L. Crassidis and F. L. Markley, "Unscented filtering for spacecraft attitude estimation," Journal of Guidance, Control, and Dynamics, vol. 26, no. 4, pp. 536-542, 2003.

[4] L. Sige, Z. Xiaoxin, F. Mingtian, and Z. Zhuping, "Probabilistic power flow calculation using sigma-point transform algorithm," in Proceedings of the International Conference on Power System Technology (POWERCON '06), October 2006.

[5] I. N. A. Romani and H. Abdalla Jr., Síntese de Filtros de Microondas Compactos com Respostas Elípticas e Pseudo-elípticas utilizando a Variável Transformada Z, Brasília University, Brazil, 2006.

[6] E. A. da Costa Jr. and L. R. A. X. De Menezes, Propagação de incertezas em eletromagnetismo, Ph.D. thesis, Brasília University, Brazil, 2009. 

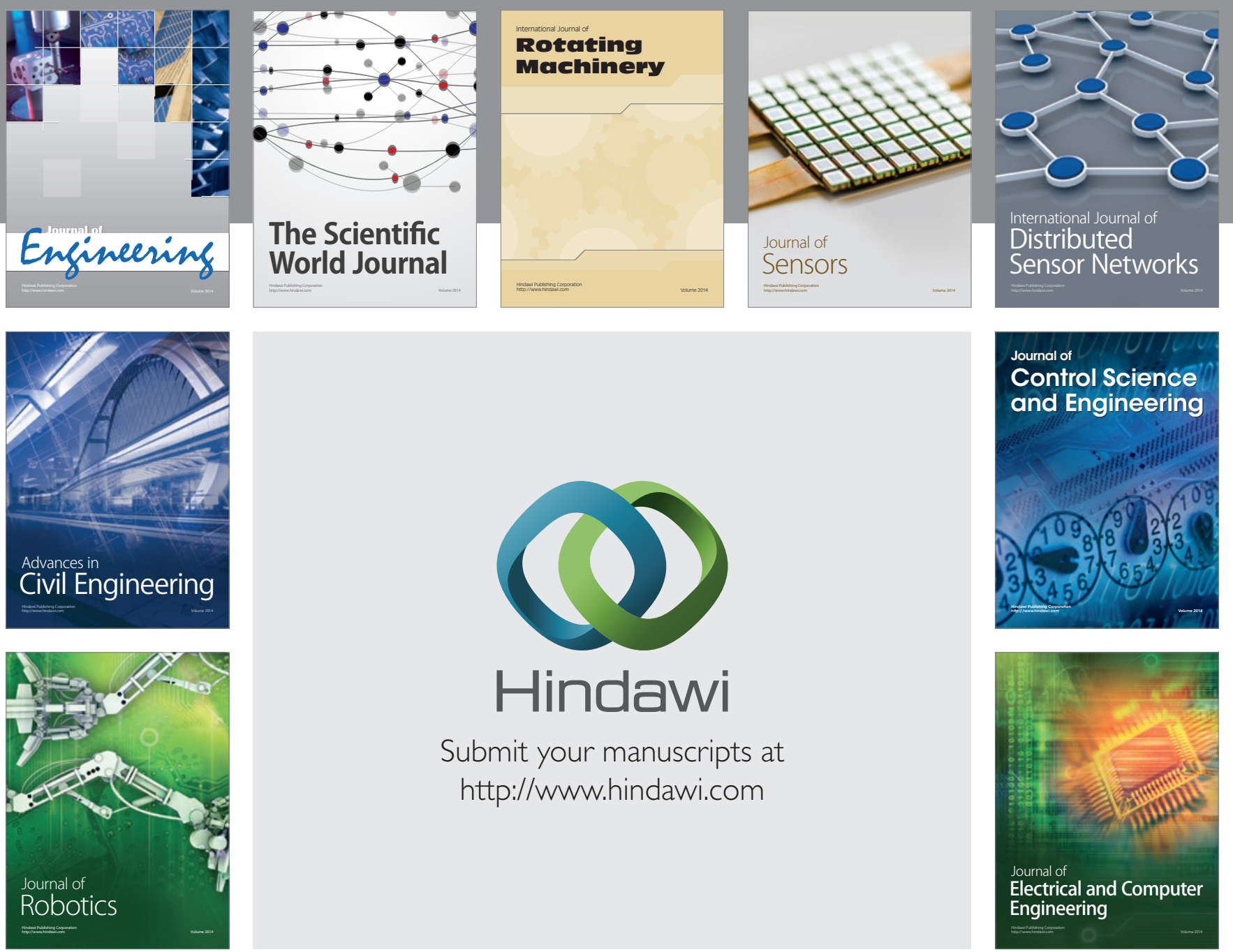

Submit your manuscripts at

http://www.hindawi.com
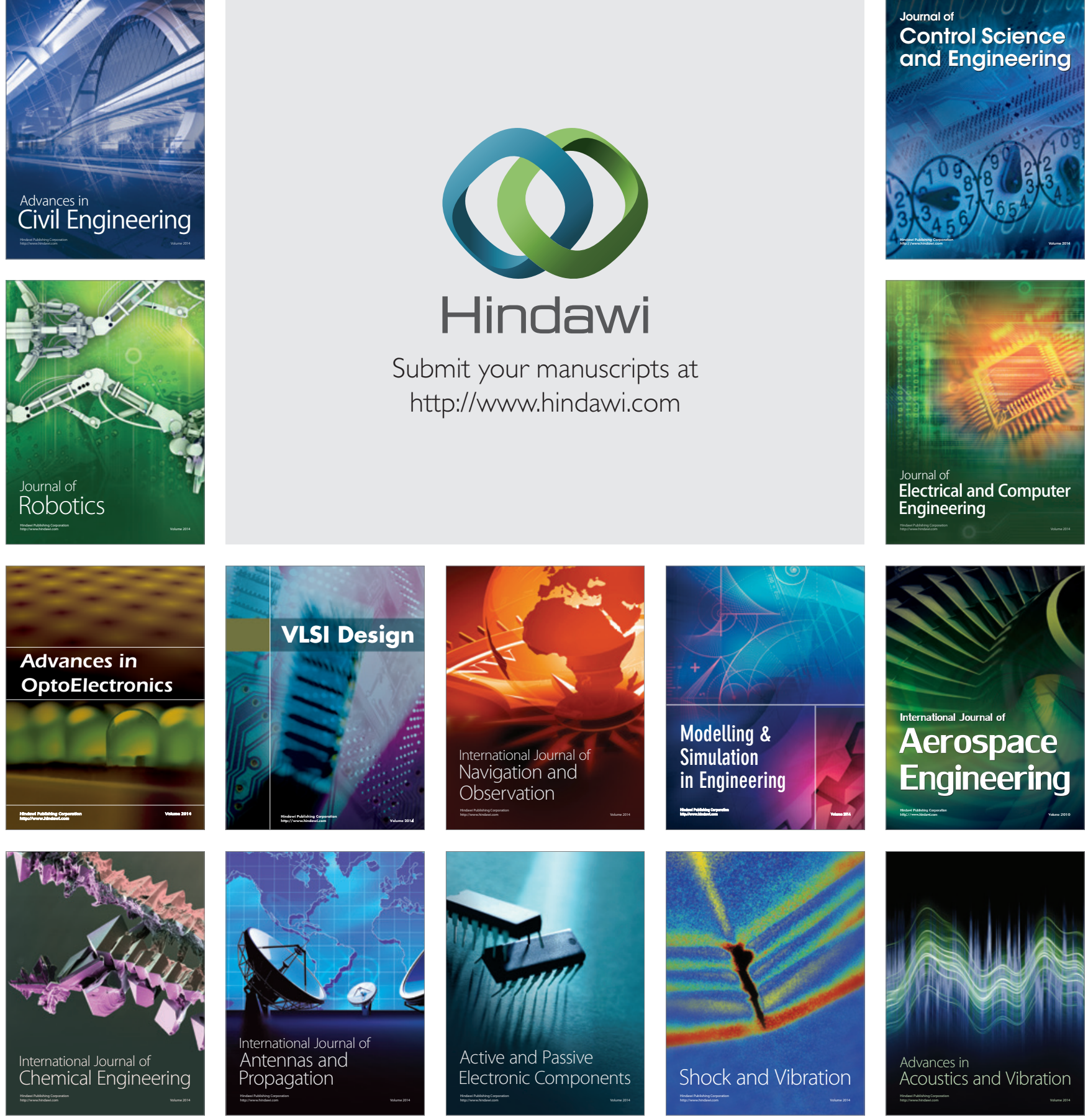